\title{
Sciendo
}

Administration, vol. 69, no. 3 (2021), pp. 1-18

doi: 10.2478/admin-2021-0018

\section{A design-led framework for engaged research: Using a design approach to understand and place the public at the core of health and social care}

\author{
Mary Galvin \\ Department of Design Innovation, Maynooth University, Co. Kildare, \\ Ireland \\ Avril Kennan \\ Health Research Charities Ireland, Digital Office Centre, 12 Camden \\ Row, Dublin 8, Ireland \\ Éidín Ní Shé \\ School of Population Health, University of New South Wales, Sydney, \\ Australia
}

\begin{abstract}
This paper offers a multi-perspective approach on the role of engaged research in health and social care. Each of the authors focuses on their individual experiences of this domain, from the perspective of an academic partner of the Health Research Board's PPI Ignite programme, a CEO of an umbrella organisation for health research charities and a researcher in design innovation, focusing on health research. The paper outlines the values which underpin public and patient involvement, as well as examples of its application as engaged research. It details how organisations like Health Research
\end{abstract}


Charities Ireland support and enable engaged research within health and social research and policy. This paper offers a framework for facilitating dialogue and response across all stakeholders in the engaged research process, illustrating the importance of engaged research and how we can further our understanding and application of it within health and social care policy by adopting a design-led approach. We argue that a design-led approach can both facilitate engaged research as well as support policymakers in the design of new policies and practices.

Keywords: Design thinking, co-design, social innovation, public and patient involvement, healthcare, social care, policy, engaged research

\section{Introduction}

The development, discussion and drafting of this paper have been undertaken during a period of unprecedented emergency in Ireland. The rapid spread of Covid-19 across the globe has instigated a radical public health response (Adalja et al., 2020; Lynch, 2020). The state has had to intervene quickly within welfare, social and health services by providing additional support to the public (Lynch, 2020). A key component of the response has been focused on addressing the crisis of public understanding (Ali \& Kurasawa, 2020). The World Health Organization (WHO) guidance on effective modes of communicating stresses the importance of educating the public about the issues involved by using the principles of trust and transparency and enabling ongoing dialogue with the community (WHO, 2005). The importance of understanding and engaging our public is central to enabling state responses within an emergency (Ní Shé et al., 2018). While communication with the public has been essential in terms of policy changes, there is much to be learned from also observing what the public can do for themselves. For example, in relation to physical distancing, self-isolation and cocooning, the online and offline behaviour of the Irish public proved vital for the adoption and acceptance of what their state was advising them to do. This emergency has pushed us to go further in our understanding of participation. By participation, we mean the dialogue between the state and public, but also how the public engage with each other and act within themselves to enable social change. This is also true for engaged research (which will be further explored as we progress across the perspectives in this paper), in that to fully enable its role within health and social care policy, we need to find a way to meaningfully incorporate all potential stakeholders. 
Defined as 'the joint working of people who are not in the same organisation to produce goods or services' (Oliver et al., 2019, p. 2), co-design brings together diverse groups, including the public and other stakeholders, to develop deeper understandings and innovate new solutions to complex problems (Hickey, 2018). It sits under the umbrella of collaborative research practices alongside 'co-creation, stakeholder and public engagement, participation/involvement and integrated knowledge' (Oliver et al., 2019, p. 1). These terms are often used interchangeably by different disciplines, particularly within health. A design approach can remove the noise around the terminology of collaborative research practices (Fransman, 2018), providing one common language for the dialogue between the state and public, which seeks to bring about social change through participation, empowerment, understanding and, by extension, truly engaged research. It can do so without ever undermining or replacing the values of collaborative research practices, as design is not about 'us-versus-them' or even 'us-on-behalf-of-them' - for the design thinker, it has to be 'us-with-them' (Brown \& Katz, 2011, p. 382).

The main aims of the multi-perspective approach of this paper are to: address engaged research from the perspective of embedding public and patient involvement (PPI) within an Irish university, further our understanding of PPI by illustrating its merits and values at both research and policy level from the perspective of an umbrella organisation for health research charities, and finally combine the methods, values, stakeholders and processes used within PPI to offer a design-led response and therefore a discipline-agnostic approach to understanding, implementing and evaluating engaged research within health and social care policy.

\section{Engaged research from a university perspective}

In relation to engaged health-related research within university settings, it is essential to address PPI, which has fundamentally changed the health research landscape over recent years. While there continues to be a significant emphasis on advancing our understanding of PPI in health and social care (Ní Shé et al., 2018), our definition of PPI stems from the UK National Institute for Health Research (n.d.) advisory group INVOLVE as 'research being carried out with or by members of the public rather than to, about or for them'. It is important to understand the difference in language as often the terms 'involvement', 'engagement' and 'participation' can be used 
interchangeably but have very different meanings, as outlined in Figure 1.

\section{Figure 1: Understanding the differences between involvement, engagement and participation}

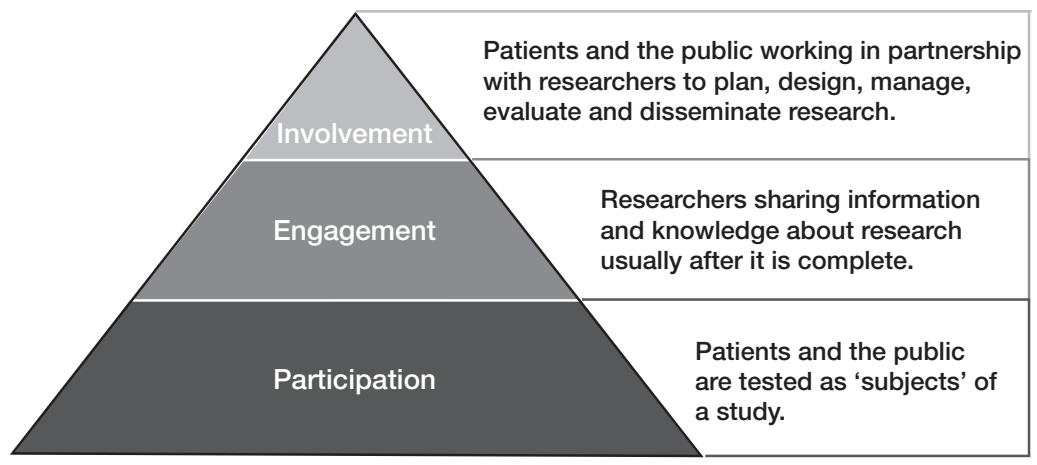

Source: Guy's and St Thomas' Biomedical Research Centre (n.d.).

Globally, we have seen significant growth and support infrastructure for PPI. In Canada the Institute for Health Research has developed a strategy for patient outcome research. Here in Ireland two funders, the Health Research Board (HRB) and the Irish Research Council, came together to launch the PPI Ignite Awards in 2017, which are focused on enabling institution-wide PPI responses within universities. Initially, five universities received funding under this scheme: University College Dublin, Dublin City University, Trinity College Dublin, University of Limerick and National University Ireland, Galway. In 2021 this scheme was expanded to develop the National PPI Network. Along with the five original partners, University College Cork and the Royal College of Surgeons have joined the network, as well as ten national and fifty-four local partner organisations (HRB, 2021). As PPI becomes more embedded as a core activity in many national and international funding calls, the evaluative literature has shifted to capture its impacts (Brett et al., 2014; Burton et al., 2015; Ocloo \& Matthews, 2016; Staley, 2015). These shifts have resulted in new demands to support, develop and sustain long-term reciprocal partnerships. Key to this are strategic initiatives designed to enhance the collaborative capacity skills of researchers, the public and those working within the health and social care system (Ní Shé et al., 2018), including appropriate methodologies to facilitate this collaboration. 
Within the UCD PPI Ignite programme there are rich and diverse examples of different types of PPI. At UCD Conway, a biomedical research and innovation institute, patient groups are involved in research within the areas of oncology, diabetes and blindness via their patient voice programs. These initiatives place patients centrally in discussions and decision-making that positively impact on treatment and patient outcomes (UCD Conway Institute of Biomolecular and Biomedical Research, n.d.). In another example of work in health systems and medicine, a research team focused upon co-designing a systematic approach to improving care for frail older patients within $\mathrm{St}$ Vincent's University Hospital (O'Donnell et al., 2019). The project team worked with public and patient representatives to enhance integrated care processes and service delivery for frail older patients within the hospital. The result was an acute care pathway for older people with integration between community-based primary care processes as well as secondary and primary care discharge pathways. At the core of the research process was the facilitation of a process of democratic dialogue in the development of quality improvement initiatives which were responsive to patient-centred outcomes (O'Donnell et al., 2019). This co-design process had at its core co-learning and recognition of mutual benefit.

Within the UCD PPI Ignite programme a broader focus has been on embedding PPI in UCD's research, learning and teaching activities and in its academic processes. There is a clear institutional commitment to firmly embed PPI in academic and administrative structures and processes (Ní Shé et al., 2018). A significant focus of the work in UCD PPI Ignite has been centred on overcoming barriers to the involvement of seldom heard voices - a term defined as:

rural communities, black and minority ethnic (BME) groups, gypsies and travellers, lesbian, gay, bisexual and transgender, asylum seekers and refugees and young carers. However, in reality, teenagers, employees, people with mental health issues and many others may be considered as seldom heard, due to the fact engagement may not be straightforward. (NHS, n.d.)

Other population groups may also face marginalisation and exclusion from engagement, including people with disabilities, frail older people and people in institutional settings (e.g. care homes, prisons). This indicates that existing structures, organisations and services that target 
the needs of the 'seldom heard' are not adequately enabling their voice to be heard via their current participation processes.

UCD sought to clarify what was needed to involve seldom heard voices in health and social care research (Ní Shé et al., 2019). It was found that PPI partners were often asked to enable research at the final stage, with researchers frequently coming to PPI partners for their signature instead of offering any meaningful process to design work together. As the project progressed, PPI partners were often spending significant time ensuring the project was culturally appropriate and accessible (Ní Shé et al., 2019). The review concluded that radical changes were required in areas such as communication, ongoing protocols to enable involvement, funding and clarity on data ownership. In these current times, we argue that these changes are still relevant, if not more urgent.

From the university perspective, PPI has played an invaluable role in engaged research, ensuring the involvement of patient groups as equal stakeholders in the research process, instead of as research study participants to analyse. If we leave the university campus, however, and explore public participation from the perspective of the community partner, what does engaged research look like? Is PPI as important and are there other roles the public can play in this space in relation to health and social policy?

\section{Engaged research from a community partner perspective}

This section seeks to further our understanding of PPI by illustrating its merits and values at both research and policy level from the perspective of an umbrella organisation for health research charities. Health Research Charities Ireland (HRCI) is the national umbrella organisation of charities active in medical and health research, representing over one million Irish patients and clients. Through support and advocacy, it represents the joint interests of thirty-nine members, working to improve health and prevent illness through research. Many member organisations were started by or heavily involve people affected by illness. They range from large research funding charities to small, voluntary-run patient organisations, all supporting patients nationally, through research, and often care. They span many areas of health, including rare diseases, childhood illnesses, cancer, dementia, mental health, and many forms of chronic illness and disability. HRCI's role is to support them in all their research endeavours and to act as their common voice with policymakers in all 
matters relating to health research. Both HRCI and its members have a key role to play in bringing together researchers, healthcare professionals, policymakers and patients in an effort to improve the quality of life for Irish patients. This different perspective of HRCI pushes us to explore what other roles the public can play in health and social policy and, more importantly, challenges us to find a way of supporting dialogue across these roles to ensure engaged research.

As community organisations, health research charities are relatively unique in their strong involvement in research. All are funders of research and also play a much broader role in helping to ensure that research findings influence policy and are translated into patient benefit. In recent years PPI has become very much part of the lexicon of the HRCI community, just as it has within the university context, and many of its members have eagerly adopted PPI as a natural evolution of their long-term representation of patient needs.

HRCI very actively supports PPI activities among its members, through a PPI Shared Learning Group. Discussions within this group allow for the identification of the challenges for community organisations as they both become involved in research decisionmaking processes themselves and facilitate the involvement of their patient communities. Key among these challenges is the fact that they are frequently asked to endorse research grant applications at a very late stage, without any meaningful involvement prior to that point, a problem also identified from the university perspective. Other significant issues include the fact that the cost of the time they give to research planning is often not budgeted in grant applications and that the weight of responsibility they carry as gatekeepers to the patient communities who trust them is often not fully comprehended. Among the group there are many experiences of tokenistic PPI, a failure to report back to the PPI contributors on the outcomes of involvement activities from university partners, and a lack of thought for the challenges that members of the public experience in taking time out of their lives to make a contribution, in a world with which they often have no prior experience.

One of the ways that HRCI is supporting the health research charities to achieve a more equitable involvement in research activities is through the development of a template form that their members can use to request information from researchers seeking PPI support. This form asks for details from researchers about their PPI plans and what they hope to achieve, and for information on what will be required 
from PPI contributors, and it strongly encourages discussions well in advance of deadlines. Through working closely with UCD PPI Ignite and similar groups, HRCI has been able to determine that such a form is acceptable, and even welcomed, by the research community. The partnership with UCD PPI Ignite is also helping to ensure that the very different perspectives of researchers and member organisations are being shaped into a mutually beneficial understanding of what good PPI should look like.

In addition to PPI in the process of undertaking research, it should also be central to policymaking as it pertains to the health research and healthcare in Ireland. As one of its major activities, HRCI runs the Irish Health Research Forum, which brings together all the Irish stakeholders in health research to consider key issues and identify constructive solutions at biannual meetings. By virtue of this forum being run by HRCI, patients and their representatives are always present at these discussions of national importance and their views contribute to emerging recommendations. The outcomes of the discussion on recent topics, such as data protection in health research, have been very much shaped by the involvement of patient representatives.

While PPI helps to ensure that research and related policy are more relevant and feasible, it also strengthens the evidence that underpins charity advocacy campaigns. In recognition of this, HRCI has developed guidance for charities on using and generating evidence to improve policy, including a section advising on how to partner with academics (Lynch et al., 2019).

Finally, within this section on community organisation perspectives, we share a practical example of a successful partnership between the public and charity sectors, that of the Joint Funding Scheme, run by the HRB and HRCI, which commenced in 2006. This innovative scheme facilitates the co-funding of research in any health condition between HRCI members and the HRB. At the initiation of each round of the scheme, participating charities issue a call for research project proposals to address challenges for their patient populations. Charity staff or volunteers manage an international peer-review process and, guided by this, decide which projects they wish to progress to the next stages. In addition to the obvious involvement of the charities, PPI is strongly encouraged, and often facilitated by the charities, in the development and undertaking of the projects. Selected projects are then rigorously assessed by an international panel of health research experts which is managed by the HRB and includes PPI contributors. 
The cost of successful projects is split between the charity and the HRB.

The scheme offers the opportunity for the state to leverage its investment in health research and helps to ensure that it is supporting projects which have relevance to Irish patients. It also helps build capacity in Irish research charities and to ensure that all elements of their research processes are undertaken according to the HRB's excellent funding practices. After each round of the scheme, the HRB and HRCI come together to review the process and to make improvements for next time. Over time, modifications to the scheme have brought about the possibility for charities to submit one application more than they can fund for review by the panel, to partner with other charities on applications and, for charities with very low income, to receive 75 per cent funding from the HRB for their projects.

HRCI provides a conduit for the Joint Funding Scheme and works with the HRB in all aspects of its running. It supports the charities through the multi-step process and also in their wider health research activities, all of which ultimately result in the funding and support of stronger research. To date there have been 9 funding rounds and 124 awards made, with approximately $€ 12.5$ million each of HRB and charity funding dispensed over that time. Thirty-four different charities have had projects funded through the scheme, including Alpha One Foundation, Epilepsy Ireland, the Irish Cancer Society and Diabetes Ireland Research Alliance (HRB, 2021).

Engaged research from the perspective of the community partner highlights PPI as equally important as the university perspective does, as well as pointing to the other roles that the public can play in health and social policy. It also draws attention to the damage that tokenistic PPI can have upon true involvement in engaged research. What is now required is a response to these perspectives that can include all stakeholders in a way that ensures successful PPI across all stages of the research process but also a way to ensure that research findings are translated to policymakers. We argue that this response lies within a design approach.

\section{Engaged research from a design perspective}

Successful engaged research involves the public transitioning from participation to engagement, to involvement, from being classified not just as study participants but as active partners in the research process. 
This active role of the public is further problematised with reference to research relating to seldom heard groups - that is, those whose engagement in society, aside from research agendas, has not been straightforward, particularly in relation to decision-making processes (Ní Shé et al., 2018). As Ní Shé et al. (2019) argue, radical changes are required in areas such as communication, ongoing protocols to enable involvement, funding and clarity on data ownership. These radical changes need to take place at the university level but also at the level of the PPI partner themselves, which was further illustrated by the work of HRCI in terms of the resources made available to health research charities.

As stated, HRCI acts as the interface between the health research community and the general public. It plays an important role in connecting health researchers with the public, facilitating research funding, as well as involving the charities that they represent in decision-making policies and processes in relation to health research. The addition of the HRCI perspective allows us to further explore and understand engagement but from the public's point of view. Rather than being a dichotomy between researcher and public, engaged research is a multi-layered, multi-perspective ecosystem, with each stakeholder bringing their own resources and agendas. A framework is required that includes all stakeholders, and that can embody the nuances that each brings to the research process. Aside from advocating for engaged research, how do we actually execute and identify a piece of engaged research in this ecosystem in a way that ensures all voices are heard but also that the research is efficiently executed to provide timely results and impact on health and social practice and policy?

A design-led approach includes each stakeholder in the research process, incorporating nuance and flexibility to ensure engagement across the entire ecosystem. It gives us an innovative, disciplineagnostic toolkit to use; however, it also provides a theoretical foundation that aligns itself with engagement, lived experience and PPI. The Double Diamond model is synonymous with design thinking. Originally presented by the Design Council in 2004 as a visual description of the design process, the Double Diamond is often referenced and updated to allow designers and non-designers (the latter largely influenced by the publication of Change by Design by IDEO CEO, Tim Brown, in 2011) to use design thinking in their practices and processes. 
The first diamond is situated within the problem space; the second within the solution space. The 'traditional' academic researcher is comfortable within the problem space, designing research questions, conducting primary and secondary data collection and analysis, and presenting a final research output. The solution space is unusual for the 'traditional' academic researcher in so far as the research process does not usually extend to responding to the answer of the research question in the form of product, service and/or policy. Usually the research is disseminated with the intention of informing policy. For successful engaged research to occur across the health context from university to policy levels, both spaces are required. However, in order for this to occur, a bridging between them is required, and to achieve this we need to identify the different roles the public can play.

Many will be familiar with design's role in healthcare in the traditional sense of the development of medical devices, but nowadays design is increasingly becoming commonplace in the development of healthcare practice and policy (Groeneveld et al., 2018). Simon (1988) argues that 'the intellectual activity that produces material artifacts is no different fundamentally from the one that prescribes remedies for a sick patient or the one that devises ... a social welfare policy for a state'. This relationship between health and design research is not a forced pairing. Just as health research moved from a medical model of care to a person-centred approach, design also broadened in its scope, moving from a 'trade activity' focused upon product development (Buchanan, 1992) to a discipline combining theory and practice across a variety of sectors to deal with a 'class of social system problems which are ill-formulated, where the information is confusing, where there are many clients and decision makers with conflicting values' (Churchman, 1967, p. 141). As design problems were embraced as more socially complex, design itself was embraced as a discipline to borrow and lend methodologies from and to, in order to change existing situations into preferred ones (Simon, 1988). If we return to the two previous perspectives on engaged research, what is the preferred situation?

HRCI occupies a role that lifts us out of academic research (health and design) and into a practitioner-led space. HRCI sits at the juncture between the health research community and the general public. For HRCI, there is no one specific research project on which to focus. Are the values of HRCI different from UCD in terms of engaged research? No, but there are different priorities and more 
complex participatory situations to navigate. The outcome for UCD is a research output (product), whereas for HRCI it takes a more relational output (service). What HRCI is successfully doing is identifying the stakeholders of engaged research. Stakeholder mapping is an important tool used with design thinking. It is a visual or physical representation of the various groups involved with a particular service. HRCI interacts with all stakeholders in the health context: patients, academic researchers, funding bodies, as well as policymakers. This is where design thinking becomes useful, as being able to represent these groups can allow the interplay between them to be charted and analysed. What is key to this process is that the patient or client - that is, the public - will always remain at the core of this interplay. The addition of the HRCI perspective to the university perspective on engaged research allows us to incorporate design as practice as opposed to solely as theory, encouraging us to consider the role of engaged research as a service as opposed to a product. In doing so, it allows us to move the solution space of the design thinking process.

If we take an approach to engaged research as a service rather than a product, it allows us to incorporate the perspectives of all stakeholders, removing the researcher and public dichotomy and ensuring engagement across the entire health research process and ecosystem by all relevant stakeholders. It also makes transparent the different roles that these stakeholders play. This transparency has the potential to be useful for evaluation of engaged research, as adopting an engaged-research-as-service approach will identify where and when the public enter and leave the process. In service design these are known as touchpoints. Typically, touchpoints are every contact point between a customer and a service provider. These interactions can be human-human, human-machine or machine-machine, but also take place indirectly via third parties, such as through online media and/or even physical trips to a building (Stickdorn \& Schneider, 2012). In relation to the tools of service design, we can represent these touchpoints and stages using customer journey maps, which represent the user's or patient's experience, and service blueprints, which incorporate the perspectives of the user, the service provider (be that university, HRCI, Health Service Executive or Department of Health) and other relevant stakeholders (such as funding bodies and local support groups) of the service. It should present not only the observable interactions with a service but also the behind-the-scenes operations that are often not public knowledge. The service blueprint 
is intended to be a living document, which means that it should be regularly revised. The purpose of this paper is not to describe these tools (for this, please consult Stickdorn \& Schneider, 2012). Instead, the purpose is to highlight their suitability to the engaged research process. By visually representing and making explicit each interaction the public has across the engaged research process, from hearing about a research study to being directly affected by a healthcare policy, a clearer understanding can be achieved as to how the public can be placed at the core of health and social care. Also, by using tools that are considered living documents, we can update, monitor and respond to policy changes as they occur. As illustrated in Figure 2, there are four stages in the design thinking process: discover, define, develop and deliver. The discover and define stages align with the initial stages of research - that is, trying to understand the problem to be solved so that the correct research question can be focused upon. This paper has highlighted the need for PPI at these stages. In the second diamond, stages of develop and deliver involve a shift in focus to solutions, answering the research question through co-design and prototyping of potential solutions. This whole process situates the study participant at its centre, ensuring that a final solution (research output) is never offered that is removed from the needs of the people for which it was designed. We argue that by following this method, health-related research will always remain engaged as it cannot work without participation of key stakeholders across the entire process. However, we also argue that aside from providing a framework for engaged research, design thinking can support the translation of research findings into practice and policy. Mintrom \& Luetjens (2016, p. 391) state that design thinking has 'the potential to improve problem definition, mechanism design, and implementation in policymaking', and argue that while policy implementation has long depended on the design of products and services, policymaking on the other hand is a design activity that has yet to be explored using design terms. They, along with the authors of this paper, argue that if we take this framework for engaged research - that is, how researchers can keep their study participants at the core of their work across the four stages of the design thinking process - and apply it to policy, we can ensure that governments are trying to solve the right problems facing society. Presented in Figure 2 is a design-led framework for engaged research, based upon the design thinking process and situated within the context of health and social care policy. 
Figure 2: A design-led framework for engaged research

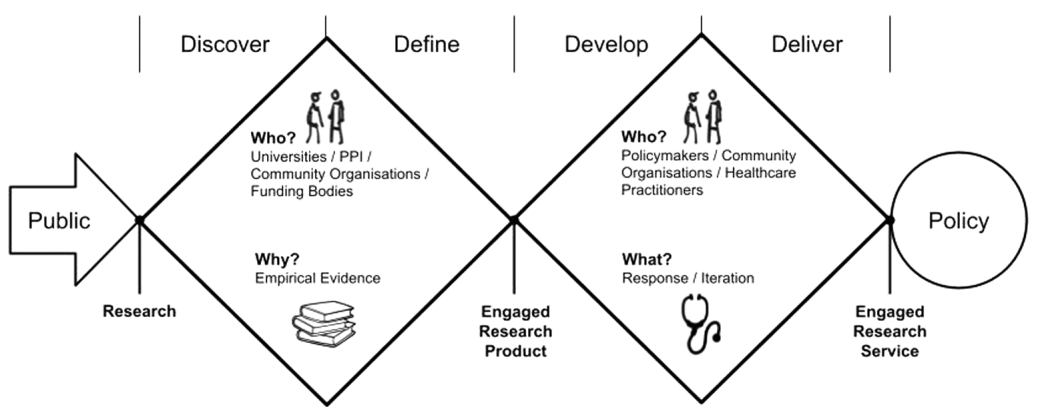

Increasingly, in the area of health, design thinking and innovation have attracted the interest of policymakers. Windrum et al. (2016) argue that this is because innovators (in particular social innovators) are better able to deliver more effective social services because of their specialist knowledge of the public's needs, ability to design new services in response to these needs, and ability to prioritise the ethical and social impacts of their innovations. Within the third sector, social innovation has been prospering in recent years. Already noted is the vital role that HRCI plays for its member charity organisations in the context of including the public in health research, policy and decisionmaking, as well as the importance of engaging with PPI partners from the university perspective. We therefore argue that private and public sectors can learn from the innovations that are already occurring within the third-sector space to incorporate engaged research into policy.

\section{Conclusion}

In recent years 'design' and 'design thinking' have become commonplace in academic discourse, with various disciplines advocating for human-centred, solution-focused approaches to problem-solving. Equally, across industry boardrooms, executive chatter has embraced design approaches as a way of strategically innovating their products and services. But, despite their transformative potential, we have not yet seen such topics embraced by public discourse. As stated previously, in 2006 design thinking was advocated as an empathetic way to understand the market environment and advance strategic management. Since then, design 
thinking has become something of a buzzword. The current popularity of design thinking, and the tension between its disciplinary origins and commercial appropriation are not the focus of this paper, but its unique characteristics, which makes it different to other means of problem-solving, are presented as a means to maintain engaged research while simultaneously translating research findings to policymakers.

However, while advocating a design-led approach, the authors also caution against the application of design-thinking methods without proper expertise. It is important to note that if design thinking is applied without a proper understanding of the values, theories and practices of doing so, we run the risk of poor execution. Many secondand third-sector organisations are now valuing the role for design and innovation within their departments and strategies (Whitham et al., 2019), with governments creating roles for designers in the development and delivery of their processes, policies and strategic innovations. This has moved government services to a co-design process away from policy program silos (Bridge, 2012). While putting the public at the core of policy interventions and involving them in policy delivery and design is not unusual for policymakers, it is difficult to execute such an ideal (Lindquist et al., 2013). By prioritising engaged research, using a design-led approach, we can bridge the theoretical co-design processes at the initial stages of engaged research with the applied design-thinking response at the later stages, ensuring dynamic and responsive public involvement.

\section{References}

Adalja, A. A., Toner, E., \& Inglesby, T. V. (2020). Priorities for the US health community responding to COVID-19. JAMA, 323 (14), 1343-4. doi:10.1001/jama.2020.3413

Ali, S. H., \& Kurasawa, F. (2020, March 22). \#COVID19: Social media both a blessing and a curse during coronavirus pandemic. Retrieved from http://theconversation.com/covid19-social-media-both-a-blessing-and-acurse-during-coronavirus-pandemic-133596 [26 July 2021].

Brett, J., Staniszewska, S., Mockford, C., Herron-Marx, S., Hughes, J., Tysall, C., \& Suleman, R. (2014). Mapping the impact of patient and public involvement on health and social care research: A systematic review. Health Expectations: An International Journal of Public Participation in Health Care and Health Policy, 17 (5), 637-50. https://doi.org/10.1111/ j.1369-7625.2012.00795.x 
Bridge, C. (2012). Citizen centric service in the Australian Department of Human Services: The department's experience in engaging the community in co-design of government service delivery and developments in egovernment services. Australian Journal of Public Administration, 71 (2), 167-77. https://doi.org/10.1111/j.1467-8500.2012.00763.x

Brown, T., \& Katz, B. (2011). Change by design. Journal of Product Innovation Management, 28 (3), 381-3. https://doi.org/10.1111/j.1540-5885.2011. 00806.x

Buchanan, R. (1992). Wicked problems in design thinking. Design Issues, 8 (2), 5. https://doi.org/10.2307/1511637

Burton, L. J., Shé, É. N., \& Olliver, S. (2015). Embedding an empowerment evaluation framework to create a 'win-win' engaged research partnership with communities. Retrieved from https://www.semanticscholar.org/paper/ Embedding-an-empowerment-evaluation-framework-to-a-BurtonSh\%C3\%A9/6120239f00bc07911eaa0da1a56398e01fc5f1b8 [16 June 2021].

Churchman, C. W. (1967). Guest editorial: Wicked problems. Management Science, 14 (4), B141-B142.

Fransman, J. (2018). Charting a course to an emerging field of 'research engagement studies': A conceptual meta-synthesis. Research for All, 2 (2), 185-229. https://doi.org/info:doi/10.18546/RFA.02.2.02

Groeneveld, B., Dekkers, T., Boon, B., \& D’Olivo, P. (2018). Challenges for design researchers in healthcare. Design for Health, 2 (2), 305-26. https://doi.org/10.1080/24735132.2018.1541699

Guy's and St Thomas' Biomedical Research Centre. (n.d.). Patient and public involvement toolkit. Retrieved from http://www.guysandstthomasbrc. nihr.ac.uk/researchers/patient-public-involvement-advice/ppi-toolkit/ [29 April 2020].

Health Research Board. (2021). Health Research Board expands opportunity for the public, patients and carers to be involved in health research. Retrieved from https://www.hrb.ie/news/press-releases/single-press-release/article/ health-research-board-expands-opportunity-for-ppi/ [31 May 2021].

Hickey, G. (2018). The potential for coproduction to add value to research. Health Expectations, 21 (4), 693.

Lindquist, E. A., Vincent, S., \& Wanna, J. (2013). Putting citizens first: Engagement in policy and service delivery for the 21st century. Canberra: ANU Press.

Lynch, B., Dunne, C., Aherne, F., \& Kennan, A. (2019). Evidence for advocacy: A practical guide. Retrieved from https:/hrci.ie/wp-content/uploads/ 2019/09/6013-Evidence-for-Advocacy-Guide_WEB.pdf [16 June 2021].

Lynch, P. (2020, March 22). We need a stronger health service - and not just in times of crisis. Retrieved from https://www.businesspost.ie/insight/we-needa-stronger-health-service-and-not-just-in-times-of-crisis-c44b889e [29 April 2020]. 
Mintrom, M., \& Luetjens, J. (2016). Design thinking in policymaking processes: Opportunities and challenges, Australian Journal of Public Administration, 75 (3), 391-402. https://doi.org/10.1111/1467-8500.12211

National Institute for Health Research. (n.d.). What is public involvement in research? Retrieved from https://www.invo.org.uk/find-out-more/what-ispublic-involvement-in-research-2/ [29 April 2020].

NHS. (n.d.). Working with seldom heard groups. Retrieved from https://www.england.nhs.uk/participation/resources/involveseldom-heard/ [29 April 2020].

Ní Shé, É., Davies, C., Blake, C., Crowley, R., McCann, A., Fullen, B., ... \& Kroll, T. (2018). What are the mechanisms that enable the reciprocal involvement of seldom heard groups in health and social care research? A rapid realist review protocol [version 1; peer review: 3 approved]. Retrieved from https://hrbopenresearch.org/articles/1-7 [29 April 2020].

Ní Shé, É., Morton, S., Lambert, V., Ní Cheallaigh, C., Lacey, V., Dunn, E., Loughnane, C., ... \& Kroll, T. (2019). Clarifying the mechanisms and resources that enable the reciprocal involvement of seldom heard groups in health and social care research: A collaborative rapid realist review process. Health Expectations: An International Journal of Public Participation in Health Care and Health Policy, 22 (3), 298-306. https://doi.org/10.1111/hex.12865

Ocloo, J., \& Matthews, R. (2016). From tokenism to empowerment: Progressing patient and public involvement in healthcare improvement. BMJ Quality \& Safety, 25, 626-32.

O'Donnell, D., Ní Shé, É., McCarthy, M., Thornton, S., Doran, T., Smith, F., O'Brien, B., ... \& Cooney Marie, T. (2019). Enabling public, patient and practitioner involvement in co-designing frailty pathways in the acute care setting. BMC Health Services Research, 19 (1), 797. https://doi.org/ 10.1186/s12913-019-4626-8

Oliver, K., Kothari, A., \& Mays, N. (2019). The dark side of coproduction: Do the costs outweigh the benefits for health research? Health Research Policy and Systems, 17 (33), 1-10. https://doi.org/10.1186/s12961-019-0432-3

Simon, H. A. (1988). The science of design: Creating the artificial. Design Issues, $4(1 / 2), 67-82$.

Stickdorn, M., \& Schneider, J. (2012). This is service design thinking: Basics, tools, cases (1st ed.). New Jersey: Wiley.

Staley, K. (2015). 'Is it worth doing?' Measuring the impact of patient and public involvement in research. Research Involvement and Engagement, 1 (6).

UCD Conway Institute of Biomolecular and Biomedical Research. (n.d.). The patient voice in health research. Retrieved from https://www.ucd.ie/conway/ engagement/forpublicpatients/ [29 April 2020].

Whitham, R., Pérez, D., Mason, K., \& Ford, C. (2019). Realising the value of open innovation in policy making: Equipping entrepreneurs for valuation 
work. The Design Journal, 22 (sup1), 189-201. https://doi.org/10.1080/ 14606925.2019.1595857

Windrum, P., Schartinger, D., Rubalcaba, L., Gallouj, F., \& Toivonen, M. (2016). The co-creation of multi-agent social innovations: A bridge between service and social innovation research. European Journal of Innovation Management, 19 (2), 150-66. https://doi.org/10.1108/EJIM-052015-0033

World Health Organization. (2005). WHO outbreak communication guidelines. Retrieved from https://www.who.int/csr/resources/publications/WHO_ CDS_2005_28en.pdf [16 June 2020]. 